\title{
Filigrane
}

Écoutes psychothérapiques

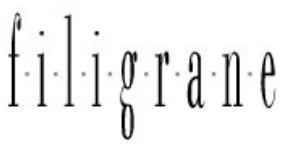

\section{Perdre de vue l'homophobie. Une ouverture sur un temps autre}

\section{Anthony Bourgeault}

Volume 21, numéro 1, printemps 2012

Psychanalyse et engagement

URI : https://id.erudit.org/iderudit/1012881ar

DOI : https://doi.org/10.7202/1012881ar

Aller au sommaire du numéro

Éditeur(s)

Revue Santé mentale au Québec

ISSN

1192-1412 (imprimé)

1911-4656 (numérique)

Découvrir la revue

Citer cet article

Bourgeault, A. (2012). Perdre de vue l'homophobie. Une ouverture sur un temps autre. Filigrane, 21(1), 39-61. https://doi.org/10.7202/1012881ar

\section{Résumé de l'article}

Ce texte a été remanié à partir d'une conférence prononcée au Colloque international : éducation et homophobie, une discrimination dans le système éducatif, présenté à l'Université de Franche-Comté, en juin 2010. Ainsi, il était d'abord adressé à un auditoire de scientifiques, d'intervenants et de militants, engagés dans un projet de lutte contre la violence homophobe, et pour la plupart étrangers à une pratique clinique et à la psychanalyse. Mettant à profit sa jeune expérience clinique et la psychanalyse telle qu'elle est pour lui vivante, l'auteur s'attèle au défi d'ouvrir ce projet de lutte contre l'homophobie à la nécessité d'un travail psychique, dans le courant duquel c'est un lien à l'autre qui se transforme et qui gagne en hospitalité. La nécessité de ce travail psychique prend son sens avec le postulat psychanalytique de l'inconscient, qui incite à considérer une intensité inconnue qui insisterait en creux dans ce qui se manifeste comme homosexualité et comme homophobie. Une écoute qui perde de vue cette homosexualité et cette homophobie manifestes oeuvre à engager la réceptivité de la parole de l'être humain à une intensité qui n'a pas fini d'y frayer sa voie. Peut alors lentement s'opérer le passage d'un agir-violence et d'un subir-violence vers une pensée accueillant la chose infantile qui fait violence.
Ce document est protégé par la loi sur le droit d'auteur. L’utilisation des services d'Érudit (y compris la reproduction) est assujettie à sa politique d'utilisation que vous pouvez consulter en ligne.

https://apropos.erudit.org/fr/usagers/politique-dutilisation/ 


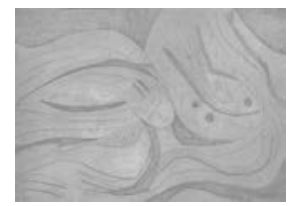

\section{Perdre de vue I'homophobie. Une ouverture sur un temps autre}

\section{Anthony Bourgeault}

Ce texte a été remanié à partir d'une conférence prononcée au Colloque international: éducation et homophobie, une discrimination dans le système éducatif, présenté à I'Université de Franche-Comté, en juin 2010. Ainsi, il était d'abord adressé à un auditoire de scientifiques, d'intervenants et de militants, engagés dans un projet de lutte contre la violence homophobe, et pour la plupart étrangers à une pratique clinique et à la psychanalyse. Mettant à profit sa jeune expérience clinique et la psychanalyse telle qu'elle est pour lui vivante, I'auteur s'attèle au défi d'ouvrir ce projet de lutte contre I'homophobie à la nécessité d'un travail psychique, dans le courant duquel c'est un lien à l'autre qui se transforme et qui gagne en hospitalité. La nécessité de ce travail psychique prend son sens avec le postulat psychanalytique de l'inconscient, qui incite à considérer une intensité inconnue qui insisterait en creux dans ce qui se manifeste comme homosexualité et comme homophobie. Une écoute qui perde de vue cette homosexualité et cette homophobie manifestes œuvre à engager la réceptivité de la parole de l'être humain à une intensité qui n'a pas fini d'y frayer sa voie. Peut alors lentement s'opérer le passage d'un agirviolence et d'un subir-violence vers une pensée accueillant la chose infantile qui fait violence.

Cette invention est le produit de deux - faut-il dire deux pensées? deux inconscients? deux appareils psychiques? - qui entrent en résonance. Absence donc d'un objet observable.

J.B. Pontalis, Fenêtres

ans le titre du présent essai, il est question de perdre de vue l'homophobie. Dans un même mouvement, il me semble qu'il pourrait être question de perdre de vue l'homosexualité, tellement ces deux termes (homophobie et homosexualité) apparaissent imbriqués l'un dans l'autre, tellement ils apparaissent réagir l'un à l'autre. 
Dans le dictionnaire des cultures gays et lesbiennes, Borrillo $(2003,255)$ définit ainsi l'homophobie: «la peur de l'homosexualité et le mépris envers les gays et les lesbiennes ou ceux qui sont présumés l'être», «le rejet de l'homosexualité [et] l'hostilité systématique à l'égard des homosexuels", "une manifestation arbitraire qui consiste à désigner l'autre comme inférieur ou anormal [...] l'homosexuel [étant] désigné encore trop souvent par la norme sociale comme bizarre, étrange ». En écho à cette définition de l'homophobie, Éribon $(2003,451)$ écrira, dans le même dictionnaire, que

La difficulté à faire face à l'effet de destin que produit la découverte de son homosexualité, la crainte d'être rejeté par la famille, [...] l'extraordinaire hostilité sociale [...], et même dans certains pays, la répression policière et judiciaire, sont autant de facteurs qui conduisent nombre d'adolescents à tenter de mettre fin à leurs jours.

Or, on s'expose à la déstabilisation si on juxtapose une récente proposition de la psychanalyste Silvia Bleichmar $(2010,50)$ à ces considérations sur l'intolérance à l'homosexualité et sur le trouble désespéré par lequel certains découvriraient une sexualité homosexuelle. Selon son hypothèse:

Le moi $[\ldots]$ ne peut s'empêcher de juger «homosexuelles» [ou hétérosexuelles, écrira-t-elle ailleurs, pour d'autres cas de figure] de nombreuses motions désirantes qui ne sont en elles-mêmes ni homosexuelles ni hétérosexuelles, du fait qu'elles se sont constituées en dehors de la différence que marque la logique identitaire de la différence des sexes.

La psychanalyste mentionne entre autres la possibilité qu'un désir de fusion avec l'autre soit confondu, par le moi, avec des désirs érotico-génitaux pour un individu du même sexe. Elle donne aussi l'exemple d'une recherche illimitée de protection qui pourrait être traduite comme contrainte de soumission homosexuelle. Ainsi, elle conçoit que la traduction de mouvements psychiques en termes d'homosexualité puisse laisser insuffisamment élaboré leur caractère infantile, irréductible à quelque identité sexuelle.

Le mode interrogatif me paraît de mise pour rendre compte de certaines implications, peut-être choquantes, qu'aurait cette proposition psychanalytique sur le problème qui nous occupe aujourd'hui: l'homophobie et l'homosexualité qui, parfois, se vit dans l'abattement. En effet, comment comprendre l'homophobie si l'homosexualité qu'elle met dans le trouble, si 
l'homosexualité qui devient son bouc émissaire, si l'homosexualité dont la découverte s'accompagnerait parfois du sentiment d'être damné, n'est pas que de l'homosexualité? Comment comprendre l'homophobie si l'homosexualité, à laquelle on suppose généralement qu'elle réagit, est plutôt la face manifeste d'une intensité encore plus immaîtrisable, en-deçà de ce qui peut se résoudre en une identité, une intensité de l'ordre de l'inconscient? En-deçà ou au-delà de ce qui se pense si facilement comme homophobie ou comme (homo)sexualité, y a-t-il autre chose qui insiste et qui n'a pas encore été éprouvé?

Il n'est bien sûr pas question de dénier l'existence de l'homophobie ou de postuler que l'homosexualité n'existe pas. Plutôt, il s'agit d'envisager que le problème manifeste de l'homophobie, de même que l'enjeu existentiel autour de l'acceptation/non-acceptation de l'homosexualité, la sienne ou celle d'autrui, soient excédés, voire déterminés, par une intensité inconnue, plus difficile à être représentée et éprouvée, une intensité infantile ignorante des catégories sexuelles homo-/hétéro-.

La proposition de Bleichmar ne me paraîtrait guère aussi inspirante si elle ne faisait écho à ma propre expérience de thérapie auprès d'un patient adolescent, rencontré dans le cadre d'un internat clinique. Sa volonté à clarifier son orientation sexuelle était un de ses principaux motifs de consultation. En offrant une écoute autre, une écoute de l'inconscient, aux souvenirs qu'il rééditait, des souvenirs parfois entachés d'homophobie et manifestement préoccupés par un enjeu homosexuel, autre chose que le litige homosexuel/homophobe est venue à nous apparaître. La communication au patient de ce que cette oreille autre entendait a d'ailleurs permis d'apaiser significativement la tension hostile qui envahissait alors les devants de la scène thérapeutique. Elle a aussi favorisé la mise en contact du patient avec ce qui le remuait intérieurement mais avec quoi il semblait encore étranger.

\section{I. À perte de vue, l'inconscient}

Le présent exposé clinique se lit comme une forme de témoignage. Selon Agamben (1998), tout témoignage résonne avec une lacune. En creux dans le présent témoignage, la lacune est peut-être même plurielle. Elle condenserait ce qui n'est pas inscrit dans la parole du patient au moment de chaque séance, ce qui ne s'enregistre pas dans mon écoute de lui, ce qui, après-coup, continue à me fréquenter comme de l'impensé, voire comme un impensable. La lacune provoque, mais aussi complique, l'écriture du temps.

Dans une perspective psychanalytique, cette lacune renvoie plus radicalement à l'origine actuelle du témoignage, à un excès qui ne se réduit pas à 
l'événement chronologiquement repérable que l'être humain croit cerner, et dont un des destins possibles est celui de faire raconter.

L'actuel, c'est ce qui n'est pas inscrit dans une chronologie, ce qui n'est pas de l'ordre du passage du temps, mais ce qui gît sous le plan chronologique comme générateur d'histoire lui-même non historisable. (Scarfone, 2000, 160)

Situer dans un rapport avec l'actuel la lacune qui se présente avec le témoignage, c'est du même coup insister sur l'acte, ou l'Agieren, que répète le témoignage. L'Agieren (l'agir ou l'acte) correspond à ce qui est toujours déjà perpétré par l'être humain et pour quoi la conscience arrive trop tard. Bien qu'après-coup, la conscience puisse chercher à justifier et à rationaliser l'acte, une étrangèreté, à propos de laquelle le sujet n'est pas en mesure de délibérer, persiste et n'acquiert pas une marque temporelle (Scarfone, 2007). Souligner l'actualité en chair dans le témoignage est ainsi une façon de reconnaître qu'à même les mots qui réalisent la temporalisation, une matière à expérience est conservée dans une intemporalité. Rolland présente cette intemporalité dans son intimité avec l'inconscient:

[...] rien de ce qui s'est passé, dont l'inconscient porte la trace (autoérotisme, excitation, traumatisme lié à l'environnement) ne s'est passé pour le moi, seul acteur d'une histoire et d'une temporalité subjective. Au contraire, ce qui s'est passé, et qui a organisé un inconscient, un ça, et qui ne s'est pas passé pour le moi, se passe toujours indéfiniment, répétitivement. (Rolland, 1998, 214)

Ainsi, qui témoigne dit, par sa parole et par ses souvenirs: cela est, cela se passe, cela s'est passé. Dans un même mouvement, mais en creux dans le témoignage, il semble aussi qu'insiste quelque chose qui soit là sans s'être passé, quelque chose ayant lieu sans avoir été éprouvé, une lacune. Quelque chose: c'est-à-dire une pensée, une connexion, un fantasme, un affect, en tout cas ce qui demeure absorbé dans une "langue qui [est] déjà là mais [qui] demeur[e] déshabitée, comme morte» (Rolland, 2006, 20). Quelque chose, encore: l'énigmatique possibilité d'une vitalité psychique qui maraude la parole manifestement prononcée et écoutée. Dans le cas du présent témoignage et des situations cliniques sur lesquelles il fait retour, on pourrait couper court le problème et compléter la phrase de cette façon: cela qui s'est 
passé... est l'identification et le rejet de l'homosexualité. Néanmoins, des questions demeurent: qu'est-ce qui, pour le moi, ne s'est pas encore passé lorsqu'on se souvient de ces événements repérables chronologiquement et identifiables à l'homophobie? Quoi d'autre que ces événements en tant que tels pousserait au souvenir? Quelles possibilités de sens pourraient révéler une parole dans laquelle de l'homosexualité est identifiée et rejetée?

Ces questions s'établissent à la condition de postuler une compulsion de représentation - le concept est théorisé par Rolland $(1998,2006)$ - à l'œuvre dans cette parole, une compulsion à remémorer qui travaille dans un sens contraire à l'oubli du quelque chose dans l'inconscient. Compte tenu de l'attraction robuste de la chose inconsciente dans un état d'impensé, celle-ci ne se manifeste jamais telle quelle dans la parole. Plutôt, la compulsion à la représentation tire la chose inconsciente de son bruit en en recueillant les effets dans la parole: celle-ci devient la substance dans laquelle ça agit, le lieu vers lequel l'agieren est détourné. Témoigner de la lacune, ou penser l'inconscient, revient alors à poser la question (à entrées multiples) : qu'agit la parole? quel acte s'y réalise? Et, en filigrane à ces interrogations : comment cette parole agit-elle sur le discours intérieur de celui à l'écoute?

J'invoquerais ici un exemple clinique, rapporté par Gantheret (1992). Il présente le fragment de la cure d'une patiente qui, séance après séance, ne cessait de parler de sa mère. Répétition faisant, l'analyste en vint à remarquer que la figure maternelle était la principale figure que les récits de l'analysante donnaient à voir. En conséquence, l'analyste s'aperçut que ses pensées à lui étaient happées par l'image de la mère. Cette dernière était celle vers qui son regard se fixait constamment, laissant dans l'ombre l'être de la patiente. Ainsi, suite à l'évocation d'un rêve, l'analyste en vint à lui interpréter: «Ici c'est moi qui ne vous regarde pas, qui regarde en direction de votre mère, sans cesse évoquée» (Gantheret, 1992, 104). Je renvoie le lecteur à l'article pour une discussion plus approfondie du cas. Néanmoins, je retiens pour le présent exposé qu'une attention à ce que la parole non pas désigne mais met littéralement en acte (une attention non pas à tel ou tel contenu se référant à la mère, mais à l'acte de parole par lequel la mère est l'aimant de tant de contenus) a favorisé l'émergence d'un dénouement de la parole, immobilisée sur la mère indifférente. Alors, le corps de la parole est devenu disponible pour penser du désir (celui de la patiente, mais aussi celui de la mère) là où il semblait n'y avoir qu'un désert.

Toujours en lien avec ces interrogations à propos du détournement de l'agieren vers la parole, j'aurais tendance à considérer les analogies (la reprise 
de mots, de thèmes, d'idées) qui se déploient à travers les associations du discours, comme autant d'actes ayant lieu à même la parole. En effet, celles-ci apparaissent dans le mouvement de la langue, sans être voulues, et possiblement à l'insu de celui qui parle. Les analogies sont là, diffractées à travers les souvenirs, les impressions, les pensées partagées, et quoi d'autre de dicible, néanmoins attenant à l'inconscient refoulé et à du désir. Les discutant longuement et les illustrant généreusement, Rolland conçoit d'ailleurs les analogies comme autant de brèches pouvant ouvrir sur «le sens encore absent de ce qui anime [la parole] » (Rolland, 2006, 98), un sens vivifié par la sexualité inconsciente. Encore un exemple: une patiente rapporte un rêve dans lequel son père lui donne à boire un sirop épais, puis un second rêve, dans lequel elle boit l'apéritif en compagnie des voisins avec qui elle s'est réconciliée. L'analyste lui fait observer "la même idée de boire, dans les deux rêves" (Rolland, 2006, 131). La patiente pense alors à un ancien rêve dans lequel elle buvait une boisson, avec l'analyste, sous une lumière sensuelle. Un fantasme de désir, impliquant l'analysante et l'analyste, en vint à être élaboré. Qu'on porte, au moment opportun, ces analogies à l'attention de l'être parlant et que celui-ci se laisse exciter par elles, et des connexions inusitées risquent bien de se former, élargissant les possibles de la parole.

Par ailleurs, cet auteur envisage un autre type de répétition participant d'un processus représentatif, mais qui serait cette fois l'effet de l'inconscient non refoulé et de la «mystérieuse sensorialité dont, sous sa langue, et par là même invisible, Psyché est pleine» (Rolland, 2006, 149). Cette répétition peut s'exprimer avec des mots, mais la parole qui les prononce apparait plate (ou trop pleine), dépourvue d'une lacune ou d'une négativité qui permettent le jeu interprétatif. Dans l'exemple clinique qu'il rapporte, cette répétition concerne la compulsion de la patiente à investir perceptivement, de façon sélective (une sélectivité commandée par l'inconscient), certains événements de la réalité extérieure autour du thème d'un écoulement qui serait à surveiller: celui des eaux de ses voisins, celui de sa chaudière, celui des pertes vaginales de sa fille. «J'y entends une plainte et de la douleur » (Rolland, 2006, 143) écrit le psychanalyste, évoquant le discours intérieur qui accompagne son écoute et dans lequel se construit une représentation d'affect (la texture endolorie d'une scène interne) là où il n'y avait que des percepts (captivés par le monde extérieur et ses écoulements objectifs). Une pensée émue est extraite ${ }^{1}$ depuis une sensorialité actuelle.

L'inconnu des mots, l'énigmatique chair qui les entoure et qui excède leur contenu manifeste, est ainsi susceptible de se révéler, au détour de 
l'analogie. Gantheret $(1996,109)$ rend bien compte de cette surprise qui, parfois, arrive avec l'analogie. Il la définit en ces termes:

L'analogie est [...] mouvement, d'écart et d'ouverture par rapport aux mots qui ne diraient que ce qu'ils disent, et ne seraient que les tenant-lieu des choses qu'ils évoquent. L’analogie est la mise en contact de deux mots, deux signifiants, si l'on veut, censés parler de la même chose, et voici que cette proximité, loin de faire inutile redondance, ouvre sur l'inouï, qui est la chair même des mots.

Les exemples précédents suggèrent la possibilité qu'en certains instants, les mots s'affranchissent de leur attachement mélancolique pour la chose que l'on croit qu'ils désignent uniquement. Et, dans l'éloignement des mots avec la chose qu'ils dénotent, vient à s'incarner ce qui n'a pas encore eu lieu pour le moi.

Aussi, les exemples précédents soutiennent l'idée que cette révélation de l'inouï engage la réceptivité d'autrui. Il faut un autre humain, oyant, à qui l'inconnu peut s'adresser. Il faut quelqu'un pour penser la lacune, pour soutenir la traversée vers la représentation, pour traduire en mots ce qu'une parole émet en acte, pour éprouver du nouveau dans la langue familière.

Dans le contexte d'une thérapie d'approche analytique, l'autre humain, c'est le thérapeute, engagé dans un dispositif d'écoute particulier. Selon les mots de Pontalis $(1988,298)$, l'écoute de l'inconscient exige une "perte dans la vue», c'est-à-dire la mise en suspens du fait historique que donne à voir la parole, de façon à ce que l'impensé qui la hante saisisse celui à qui il est raconté - et celui qui le raconte. Ce saisissement, coextensif d'un dessaisissement narcissique, est astreint au passage «d'un régime habituel [de l'écoute] où le référent est l'objet absent que la parole évoque, à un régime analytique où il est cette parole elle-même» (Gantheret, 1992, 98). Autrement dit, pour soutenir l'aperception de l'inconnu, il ne s'agit plus d'entendre le récit des souvenirs comme une succession linéaire d'événements, le long de laquelle chaque souvenir renverrait à des scènes passés que la parole se trouverait à décrire. Cette écoute habituelle du discours manifeste doit être doublée d'une écoute autre, celle-là sensible aux événements portés par la parole même, au temps où elle est énoncée. Les émois que la parole provoque dans le discours intérieur de celui qui écoute font partie de ces événements. Ce travail engage nécessairement, du côté du thérapeute, une « essentielle passivité-passibilité ${ }^{2}$ en tant que, par sa disposition à écouter, il s'offre à être affecté sur un tout 
autre plan que celui, relativement maîtrisable, de la compréhension » (Scarfone, 2010). Ainsi, le moi du thérapeute se défait nécessairement de la stabilité d'un déjà-pensé et de sa volonté à maîtriser l'autre, afin de rencontrer l'inconnu et de se laisser transformer par lui.

Dans les rencontres plus heureuses, la communication au patient de certaines pensées générées par cette écoute favorise qu’il puisse investir autrement, voire nouvellement, différentes parties de son intériorité et du monde, et qu'il se laisse approcher, avec moins de réticence, par l'étranger. Ces considérations dessinent un horizon inusité, qu'on ne finit pas de balayer.Y auraitil une disponibilité éprouvante à envisager pour une autre chose émanant de la parole qui, sur un plan manifeste, communique un contenu identifiable à de l'homophobie? Quelles scènes insoupçonnées, quels affects non vécus, quelles souffrances méconnues feraient signe ou acte dans la communication d'une insulte à caractère homophobe ou dans le récit exemplaire d'avoir été intimidé en raison d'une orientation sexuelle? Qu'adviendrait-il si nous perdions de vue, sans pour autant les dénier, l'homosexualité que, pour paraphraser Foucault, nous voulons savoir et l'homophobie que nous savons déjà arrivée? Laisserons-nous, alors, l'étranger nous regarder? Qu'imagineronsnous depuis l'inconnu des mots?

\section{II. Écoute (d'une) clinique ${ }^{3}$}

Le présent témoignage, bien que lacunaire, n'est cependant pas uniquement le constat d'un inconnaissable. Il fait aussi retour sur une expérience affective de pensée, dans laquelle mon écoute et la parole du patient se sont entrouvertes sur une intensité encore sans âme, alors qu'étaient évoqués des souvenirs manifestement préoccupés par de l'homosexualité et traversés par ce qui serait identifiable à de l'homophobie. Mon témoignage ne boucle donc pas un tracé accompli. Il pointe vers une direction, vers un lieu ou plutôt vers un non-lieu de la mémoire dont l'approche est peut-être infinie, assurément lente. Cette lenteur de la traversée, le long de laquelle on est approché par la chose inconsciente, participe certainement des limites au matériel que je peux maintenant présenter.

\section{a. Une séance durant le premier mois}

Je suis déjà passé dans le Village ${ }^{4}$ et je l'ai vu: les gais sont des obsédés sexuels. Il y en a même plein qui avaient l'air sadomaso. Ils portaient des affaires en cuir. C'était dégueux. 
Avec un certain mépris, le patient prononce cette parole dans le cours de la séance. Il se souvient d'homosexuels qu'il a croisés et qu'il juge, péjorativement, en assimilant son jugement personnel à la perception, prétendument objective, d'un voir.

Il est difficile de ne pas penser à l'homophobie lorsqu'on est témoin d'une telle déclaration. Il peut également être tentant de réagir à la perception-jugement du patient par une autre perception-jugement, du genre: "vous faites preuve d'homophobie». Il peut aussi être tentant de corriger l'aspect irrationnel de la généralisation qui affirme l'équivalence entre homosexualité et obsession sexuelle. Néanmoins, dans le travail clinique qui est le mien, la tentation de circonscrire la parole de l'autre dans une identité homophobe et la volonté d'agir promptement sur celui qui me trouble me semblent faire l'économie d'une épreuve de passibilité. Succomber trop souvent à cette tentation et à cette volonté compromet certainement l'approche de l'inconnu. Lorsque j'écoute le patient, cet inconnu n'apparaît pas aussi spontanément à mon esprit que l'idée d'homophobie, mais il participerait à déterminer sa parole. Cet inconnu ne s'appréhende qu'en laissant la parole faire son œuvre. Ainsi, il devient nécessaire de considérer cette citation dans le contexte de la parole du patient, telle qu'elle s'est déployée durant la séance.

Il commence en me parlant, avec ce qui m'apparaît comme une certaine irritation, des histoires d'amour qui surviennent entre les élèves de son école. Avec une hostilité montante, il relève le comportement des filles qui, selon ses dires, sont «profiteuses» et «amoureuses de l'amour». Certaines l'approcheraient mais il demeure méfiant: il les croit capables de le manipuler pour assouvir leur soif de romance. Néanmoins, il rapporte préférer la compagnie des filles à celle des garçons, avec qui il n'entretient généralement pas de liens.

Pas de liens? fais-je, pour relancer sa parole qui s'était abruptement éteinte et dont l'extinction perdurait.

Un silence. Puis il évoque cette fois où il s'est rendu dans le Village: «Je suis déjà passé...». Son hostilité continue de croître. Et il se souvient y avoir déjà rencontré un jeune homme, qui n'avait plus donné de nouvelles après avoir couché avec lui. Il en a tiré une conclusion qu'il exprime avec rancœur : «Le gars était juste intéressé par mon corps.»

Pendant ce temps, je suis traversé par différentes pensées. Je remarque qu'il commente les gens de son environnement mais qu'il en dit peu sur comment il se sent. Il me semble qu'il rapporte des épisodes relationnels 
possiblement humiliants sans mettre à l'intérieur des mots comment il a pu être affecté. Je suis par ailleurs frappé par le moteur d'hostilité qui ne cesse de résonner dans sa façon de porter son récit. Je remarque aussi que cette hostilité, voire cette dureté, s'est intensifiée après ma relance à propos du «pas de liens» avec les garçons. En contraste avec la dureté qu'il exprime avec son ton, ne me dit-il pas pourtant qu'il est celui de qui on pourrait abuser? C'est alors à l'intérieur de moi qu'une certaine pensée de la fragilité prend forme. En effet, une impression m'apparaît graduellement selon laquelle notre lien est précaire. Je me sens sur la corde raide d'une évaluation et j'ai la fantaisie qu'il se demande ce que je lui veux : m'emparer de son corps ou prendre soin de son âme? Je crains alors de m'y prendre maladroitement, de ne pas être bon pour lui. Puis, je redoute qu'il ne soit que de passage, qu'il ne m'aimera pas s'il m'identifie à un homosexuel. Une idée inattendue s'organise ensuite qui fait le pont entre mes éprouvés contre-transférentiels, à savoir mon inquiétude qu'il ne m'aime pas, et un thème récurrent dans ses histoires, à savoir qu'on lui préfère un scénario, romantique ou sexuel, décidé à l'avance. Je pense à Aulagnier $(1991,119)$ qui écrit que le travail analytique consiste à "permettre au sujet de trouver quelle est la question à laquelle certaines réponses espèrent en vain d'imposer silence», et je lui dis, lentement:

Vous me parlez de ces filles amoureuses de l'amour et de ce garçon qui ne s'intéresse qu'à votre corps. Comme si, peut-être, vous vous demandiez: mais qui va m'aimer moi?

Un nouveau silence. Il se détend et son ton s'adoucit. Il me communique alors son agréable surprise à entendre ce que je lui dis, une surprise qui contraste avec la peur qu'il reconnaît avoir ressentie lorsque j'ai commencé à parler. En étais-je un de plus qui allait lui faire des reproches ou la morale?

Cette parole, élaborée entre ce que je ressentais en sa présence et ce qu'il racontait en ma présence, semble être apparue comme une parole de lien entre lui et moi. Et peut-être, une parole soutenant l'élaboration d'un lien perdu ou méconnu entre lui et un autre homme. D'ailleurs, répondant à mon intervention, c'est au père qui n'a "rien voulu savoir» de lui avant sa naissance auquel il pense et qu'il évoque, pour la première fois avec moi. (Et de ce père, cet homme, qu'avait voulu savoir la mère?) Le retrait agi par le silence suivant sa déclaration d'un "pas de liens » avec les garçons intègre alors davantage la trame de son discours. Quelque chose d'un vide relationnel entre le père et lui advient à la mise en mots. Dans un même souf- 
fle, il aborde le thème de sa première mise en adoption : une mère-substitut l'a accueilli, à propos de laquelle il sera dit bien plus tard, qu'elle ne pouvait pas enfanter.

Ma lecture de l'extrait n'est certainement pas exhaustive. Néanmoins, il m'apparait que le souvenir qui s'y trouve, et dans lequel se fixe de l'homophobie, utilise le récit du patient afin que celui-ci s'approche, à l'aide de celui qui écoute, d'une intensité appartenant à un registre autre. Par exemple, cette intensité pourrait renvoyer à une blessure d'amour qui le menace, bien endeçà de la question de son orientation sexuelle, une blessure d'amour actuelle au moment où il parle, et susceptible de faire violemment retour à travers tous les temps, dans la mesure où elle n'est pas suffisamment élaborée. Peutêtre, la blessure de n'être pas aimé pour lui-même, mais à la place de quelque chose... ou de quelqu'un d'autre.

À même ce doute d'amour, s'énonçant d'abord à partir de mes éprouvés contre-transférentiels, semble également insister le désir ardent d'être aimé. Mais qui va m'aimer moi... autant que je veux l'être? reformulerais-je.

Dans la perspective de Klein (1968a, 92), ce désir d'être aimé se comprend comme l'envers d'une "culpabilité inconsciente», liée à la "peur inconsciente d'être incapable d'aimer [...] vraiment les autres». Cette peur, que la psychanalyste énonce également comme la "peur d'être un danger pour la personne aimée » résulterait d'une «incapacité à maîtriser [ses] pulsions agressives ». Bien que j'entretienne mes réserves envers la trop fréquente clarté de l'inconscient dans la métapsychologie kleinienne, je recours à cet extrait parce qu'il a le mérite de jeter un nouvel éclairage sur une partie du problème: l'inquiétude quant à l'amour reçu irait de pair avec une agressivité, ou plus largement, avec une intensité pulsionnelle culpabilisée qui n'arrive pas à se lier en fantasme.

Il me semble que cette considération autorise d'interroger la dynamique psychique et culturelle menant au projet même de la traditionnelle lutte contre l'homophobie. En cette dernière, résonne manifestement un appel à être aimé autant que la plainte de ne pas l'être en raison d'une orientation... de la sexualité. Or, cette lutte ne résulterait-elle pas aussi du destin de dimensions agressives rôdant dans l'être humain, voire du destin d'une certaine culpabilité? Par ailleurs, à qui appartiendrait ce sentiment de culpabilité, à l'origine d'un appel d'amour? Est-ce si clair? 


\section{b. Une séance durant le septième mois}

J'ai pris l'autobus. À l'intérieur, il y avait un garçon qui me traitait de fif et de noms de ce genre-là à mon ancienne école. Il disait aussi qu'il allait m'arranger le portrait à un moment donné. J'ai décidé de m’enfuir de l'autobus au premier arrêt.

À un niveau manifeste, ce souvenir traduit certainement l'inquiétude du patient de se retrouver devant un éventuel agresseur. Qu'autrui ait identifié et méprisé chez lui de l'homosexualité semble participer de son inconfort. À partir de ce souvenir relatant une scène qui a déjà eu lieu, on pourrait certainement justifier des actions auprès de ses deux protagonistes. D'une part, on pourrait vouloir confronter le garçon intimidateur, de façon à faire évoluer sa mentalité. D'autre part, après avoir reconnu le malheur qu'il relate, on pourrait chercher à outiller le patient de techniques d'affirmation afin qu'il puisse mieux faire face à ceux qui s'en prennent à lui. On pourrait aussi s'acharner à combattre les effets d'une possible homophobie intériorisée, trahie par la survenue de ce souvenir. Néanmoins, il m’apparaît que ce travail d'action sur ce qui dérange ne doit pas s'effectuer sans la contrepartie d'un se laisser prendre par ce qui trouble. Il importe de ne pas perdre l'espoir qu'une forme encore inédite puisse émerger à partir de ce dérangement. Ainsi peut s'éprouver quelque chose de l'inconnu qui détermine la parole, de prime abord choquante ou désespérante. La question demeure: qu'est-ce qui ne s'est pas encore passé et qui insiste, lorsqu'on se souvient d'événements à tonalité homophobe? À nouveau, il convient de mettre ce souvenir dans le contexte élargi des associations du patient.

Le patient se présente renfrogné ce jour-là. Il garde son manteau d'hiver tout en évoquant l'été précédent et le début de sa thérapie. Il relate son attente, avant notre premier rendez-vous. Et il se souvient, considérant qu'il allait rencontrer un thérapeute homme: «Jusqu'à la dernière minute, $\mathrm{j}$ 'avais pensé m'enfuir. » Il me parle ensuite de son quotidien, de ses déplacements dans la ville: «J'ai pris l'autobus...» Il médit sur ce garçon de l'autobus. Puis, il raconte avoir déjà lancé un dictionnaire à un garçon qui l'agaçait et qui, lui, ne l'effrayait pas. "C'est toi la tapette», lui avait-il flanqué aux oreilles. Il s'enorgueillit ensuite d'avoir commencé à faire du culturisme. Il m'explique, avec une certaine condescendance, son programme d'exercices. Et soudainement, sa voix se fragilise. Il y a un garçon, à la salle d'entraînement, qu'il trouve beau. Il ajoute, avec l'irritation de celui qui s'explique mais n'en a pas 
envie: «Quand je le vois là, je ne reste pas longtemps: il faut que je m’en aille.»

En l'écoutant, je suis saisi par la reprise du thème de la fuite qui pointe dans son rapport au beau garçon du gymnase, après être apparu en lien avec le garçon intimidateur. Je pense au texte Un enfant est battu dans lequel Freud (1919) présente un scénario fantasmatique où l'objet aimé est un objet violent, et où le désir d'être battu par une figure masculine apparaît comme un substitut pour le désir interdit d'être aimé du père. C'est d'ailleurs un genre de bataille que le patient avait entretenu quand il contre-traitait un pair de tapette en lui balançant un dictionnaire. Il m'apparaît aussi qu'une atmosphère combative recouvre certaines de nos séances. Ses mots m'assènent parfois des coups et, à l'occasion, je m'inquiète de les lui rendre, coupable de mes interprétations. Je me souviens alors être moi-même apparu sous le thème de la fuite: le thérapeute était, dans ses associations, un homme duquel s'enfuir. Les premiers moments de la séance me reviennent à l'esprit. Alors qu'il se rappelait les débuts de sa thérapie, c'est à sa possible blessure d'amour, entre autres vis-à-vis l'absence paternelle, que j'avais repensé.

Je suis donc surpris par la répétition du thème de la fuite, qui se lie d'abord à l'objet transférentiel, pour ensuite dévier vers un objet violent, puis vers un beau garçon. Le thème de la fuite semble marquer la rencontre du patient avec un autre homme, avec qui l'établissement d'une relation semble problématique, voire menaçante. Ainsi, non sans incertitude, je lui propose:

Fuir le thérapeute homme, fuir le garçon qui vous intimide, et peut-être, encore la même idée de vous enfuir, lorsqu'il est question de vous en aller du gymnase lorsque vous y croisez le beau garçon. Comme s'il y avait pour vous un danger d'être en présence d'un autre homme.

Il répond à l'idée que je lui communique en s'étonnant de ne pas y avoir pensé. Avec un ton qui semble exprimer un soulagement, il y va d'un "Vous êtes fort monsieur». Il annonce ensuite qu' «il fait chaud», tout en retirant son manteau. La réponse du patient à mon intervention n'a pas été sans me surprendre puisque par celle-ci et de façon inattendue, le patient semblait s'installer dans un rapport de proximité avec sa fragilité et avec moi. Un rapprochement aussi fulgurant était inhabituel dans notre relation.

Un silence. Puis, il enchaîne, ému : 
Je risque de me sentir comme un monstre si je pense à ce que je peux faire aux hommes.

Du monstre enragé qu'il décrit, il évoque une impulsion à dominer les hommes, à prendre sur eux le dessus, jusqu'à les écraser. Puis, il pense à sa mère d'accueil : elle aussi cherche à contrôler les hommes, elle non plus n'arrive pas à cohabiter avec eux.

L'évocation de la mère enchaîne sur une parole qui dit la monstruosité (à caractère misandre) du patient. Dans l'après-coup, et à partir de cette séquence associative, des questions émergent. Par exemple, serait-il un monstre comme la mère, dans le sens qu'une identification à celle-ci étaierait la monstruosité du patient vis-à-vis des hommes? Et/ou encore par sa monstruosité envers les hommes, protègerait-il la mère d'accueil d'en être l'objet? Voire, cette monstruosité envers les hommes le protègerait-elle lui-même d'avoir à affronter psychiquement une dévorante passion à l'adresse de la figure maternelle?

\section{Après-coup ou le prélude à une nouvelle épreuve}

Un récit clinique est un récit comme un autre. Il n'échappe pas aux méandres de la construction du souvenir. Condensations, déplacements, refoulements, mais aussi un certain tri de l'information imprègnent son élaboration.

Si mon témoignage est lacunaire, c'est aussi parce que la transparence lui manque. Mon témoignage pointe dans la direction de séances arrivées pour vrai. Certainement, il les touche. Et cela importe, dans la mesure où ces séances étayent une idée que j'essaie d'introduire: c'est-à-dire qu'une écoute analytique de l'homophobie permet d'ouvrir le problème sur des enjeux psychiques, et éventuellement sociaux, qui excèdent la violence envers l'homosexualité per se. Mais, ultimement, la vérité que propose mon témoignage n'est pas celle du mot-à-mot prononcé par le patient, ni celle d'associations libres que je rapporterais, en toute objectivité. Du moins, cela rejoint ce que suppose Smirnoff $(1977,201)$ :

Disons que l'analyste témoigne de son expérience: témoignage qui n'est pas aussi candide qu'il peut le paraître au premier abord, car il ne s'agit pas d'un enregistrement des séances, mais d'un choix délibéré de moments et de mouvements en vue d'une démonstration. La clinique, en devenant objet d'écriture, subit déjà une transmutation. En n'étant pas simple compte 
rendu [...] la matière clinique devient allégorie, remise en ordre, illustration, preuve, re-création.

De nouveau, un doute est semé quant à la temporalité de l'expérience en jeu dans le témoignage. En effet, si témoigner d'une expérience implique de la recréer, de la créer autre, de la transmuter, l'expérience qui concerne le témoignage est-elle vraiment passée, ou bien présente, sinon à venir? Est-elle arrivée, arrivant ou attendue?

Dans une perspective psychanalytique, il convient de ne pas nous en tenir à une trop évidente linéarité selon laquelle l'expérience précéderait le témoignage. Certes, mon écriture suggère que cela s'est passé avec le patient, telle ou telle chose a été pensée, dite, vécue. Par ailleurs, le fait que je témoigne trahit également un reste qui ne me laisse pas tranquille. Quelque chose ne s'est pas encore passé dans ma rencontre avec ce patient. De l'inexpérimenté n'est pas mis derrière moi. Celui-là impulse la transmutation de l'événement, que constitue ma rencontre avec l'autre, en un récit clinique. Plutôt que simple retour sur une expérience passée, le récit clinique palpite désormais comme temps de transit vers une expérience à venir. Conversant avec Michel de M'Uzan, Pontalis $(1977,7)$ interroge en ce sens le rapport du texte (de psychanalyse) à l'expérience :

Tout débat sur l'écrit, et en particulier sur l'écrit analytique, me parait présupposer une sorte de gradation: on irait de l'expérience - ici, celle de l'inconscient, du processus, de la relation transférentielle - jusqu'au pensé, au théorisé, et, de là, à l'écrit, enfin au publié. [...] Devons-nous vraiment admettre comme allant de soi cette précession du vécu sur le pensé et du pensé sur l'écrit? Je serais tenté de renverser les choses: le mouvement d'une analyse ne nous montre-t-il pas que nous allons - analysé et analyste - des mots à la chair, de l'inscrit à ce que les Anglo-Saxons nomment experiencing, du roman familial ou du mythe individuel à une vérité qui prend corps?

Une partie de la vérité de mon témoignage attend de s'incarner. Bien sûr, il a pu arriver que les mots atteignent furtivement la chair, si je me fie à l'émoi partagé, en ces moments interprétatifs auxquels je me suis précédemment référé. Toutefois, je sens bien que tant d'intensité n'a pas rencontré les mots, que tant de mots n'ont pas rencontré la chair. Ni pour le patient, ni pour moi. 
L'élaboration que je souhaite maintenant introduire ne se rendra assurément pas jusqu'au corps du patient. Et les mots qui la composent n'ont probablement pas terminé de cheminer jusqu'au mien. Ainsi, cette élaboration (et les formulations interprétatives qui la ponctuent) ne constitue pas une fin à l'histoire, inachevée. Plutôt, cherche à s'y ranimer le mouvement qui va du bruit au mot, et éventuellement, du mot à la chair. En repensant à ma rencontre avec un ancien patient, la perlaboration sert l'avenir. Elle est une étape par laquelle on transite vers de nouvelles possibilités de rencontre et vers une épreuve renouvelée de l'inconnu, dans les parages de l'homophobie et au-delà.

Les questions préalablement formulées, qui ont émergé dans l'après-coup à cette séance durant le septième mois, entament le lien que je tisse maintenant. Cournut $(1983,138$-139) reconnaît que là où le patient semble manquer de représentation et d'affect pour élaborer, le travail du psychanalyste (ou du thérapeute analytique) est alors de "présumer», de «supposer », d' "imaginer». Et, suivant cet extrait de Pontalis en conversation avec de M'Uzan (1977), peut-être aussi que là où le thérapeute s'efforce à raconter des histoires, il y a matière qui n'a pas suffisamment été vécue par lui-même. Je ne peux donc nier l'apport de ma psyché lorsque, à partir des thèmes que ses associations juxtaposent, je continue à imaginer la fugitivité et le monstrueux du patient. Le lien que je tisse n'a pu être remanié par sa parole. C'est plutôt ma parole qui est ici déjà affectée par la sienne (et par celle de sa mère d'accueil) et par l'innommable qu'elle a éveillé en moi.

Ainsi, ma rêverie m'amène à supposer un lien avec un événement brièvement évoqué par la mère d'accueil, en présence du patient, à l'occasion d'un rare entretien mené avec eux deux.

La mère d'accueil avait rapporté son départ impulsif, à l'étranger, pour une période indéterminée, alors que le patient était jeune enfant. Du même coup, elle quittait son conjoint de l'époque, qui lui lançait des ultimatums: «ou bien c'est le petit, ou bien c'est moi». L'enfant a été replacé dans une autre famille, jusqu'au retour imprévu de celle dont on pourrait dire, à la lumière des thèmes ultérieurement abordés par le patient, qu'elle s'était enfuie. D'un seul départ, elle réalisait alors plus d'une séparation (entre elle et son fils, entre elle et monsieur, entre monsieur et son fils). Le thème de la fuite s'est donc introduit dans le récit de cet événement, mais cette foisci, c'est la mère qui en est l'initiatrice. La fuite apparaît comme une solution de la mère aux tourments qui envahissent sa relation à ses propres objets. Justifiant ce départ, elle invoque la dépression qui l'affligeait alors, 
et une mort : celle de son propre père avec qui la relation avait toujours été difficile.

Le récit est raconté comme un fait divers et le patient en découvre le contenu en même temps que moi. Il est stoïque. Et je ne comprends pas pourquoi il ne crie pas, ou plutôt, pourquoi il n'y a pas l'ombre d'un cri qui paraît traverser ses pensées. Le ton dans lequel sont racontés la mort de l'un et le départ de l'autre est neutre et plat. Il semble que nous nous avancions dans les contrées du deuil raté, telle que les décrit Cournut $(1983,145)$ :

Les mots du deuil n'ayant jamais été ni dits ni appris, et les scènes n’ayant jamais été dramatisées, ce sont essentiellement les affects qui sont enterrés dans le caveau.

Le travail de Cournut suggère alors qu'un sentiment (inconscient) de culpabilité puisse faire ombre à la traversée du deuil et à l'élaboration de la perte.

Par le signe d'une dépression, évoquée en concomitance avec le décès du père et avec une double rupture, impliquant l'enfant adopté et le conjoint, on suppose le spectre de la culpabilité. Quoi de cette mère aurait tué le père? Quoi de ce père n'aurait pas survécu à la mère?

La première question pointe vers le sentiment de culpabilité, comme destin possible des enjeux pulsionnels de la mère. Elle interroge la criminalité inconsciente de l'être humain. Classiquement, celle-ci renvoie aux voux de meurtre du père, tel que Freud (1916) les expose dans Totem et tabou, et à l'amour incestueux, par rapport auquel Un enfant est battu (Freud, 1919) est un texte de référence. Elle concerne aussi l'archaïque de son avidité et de son envie, décrites de façon exemplaire par Klein (1968).

La seconde question interroge la réponse traumatique de l'environnement à la destructivité, voire plus largement à la spontanéité, de la mère - qui a aussi déjà été une enfant. De ce fait, la seconde question pointe plutôt vers le sentiment primaire de culpabilité, comme destin possible traitant les expériences agonistiques de l'être humain, confronté à la non-survivance ${ }^{5}$ de l'objet primaire et au débordement d'excitations qu'elle provoque (Roussillon, 1999). Ce sentiment primaire de culpabilité se caractérise par l'instauration, dans le psychisme, d'une illusion que Roussillon $(1999,83)$ formule en ces termes: «Je suis le mal.» Alors, se vivre comme étant le mal, voire se rendre coupable (par exemple, en malmenant un père, ou en abandonnant un enfant), permettrait à l'être humain de renverser un vécu insoutenable 
d'impuissance, le traumatisme d'avoir été passivement démuni vis-à-vis un objet, insuffisamment disponible à un échange avec soi. Ainsi, il pourrait être moins insupportable de se sentir mauvais que de se sentir impuissant, d'attaquer littéralement le père que de le trouver détruit, comme "mort». Le postulat primaire de culpabilité offre au moins le bénéfice d'une illusion de contrôle sur le mal qui arrive. Il donne une forme au malaise diffus susceptible d'habiter l'être humain, et insiste comme un sens cruellement surmoïque, colmatant le non-sens douloureux d'une excitation désorganisée et désorganisante.

Peut-être, il y a les affres de sentiment(s) de culpabilité du côté de la mère, qu'une dépression entourant la mort de son père laisse deviner. Et lorsque le patient parle de lui-même comme d'un monstre par rapport aux hommes, on entend peut-être là aussi les échos d'une violence pulsionnelle, dont l'aspect terrible - hyper culpabilisé - trahirait une culpabilité primaire gardant inconsciente l'impuissance d'avoir été laissé tomber par ses premiers objets d'investissement. Roussillon (1999) reconnaît d'ailleurs que la culpabilité primaire peut se mêler aux expériences pulsionnelles et en compliquer l'élaboration. Mon hypothèse ne s'arrête cependant pas à ces théorisations qui, en dernière analyse, font de la culpabilité (œedipienne ou primaire) une solution (ou une impasse) qui serait celle du patient. Plutôt, mon hypothèse va jusqu'à présumer que la monstruosité à laquelle le patient s'identifie, dans son rapport aux hommes qu'il fuit, puisse exprimer quelque chose d'une culpabilité actuelle du côté de la mère et de ses ancêtres. Autrement dit, la solution (ou l'impasse) du patient serait alors l'identification, et le trait sur lequel porterait celle-ci serait un enjeu de culpabilité insuffisamment élaboré dans la lignée maternelle.

De façon classique, les psychanalystes envisagent l'identification à un trait de l'objet comme une solution à la perte de celui-ci. La relation d'amour (et de haine) à cet objet se pérennise alors, dans la relation que l'être humain entretient avec lui-même - à l'intérieur de son moi. Approfondissant cette idée, Jean Cournut (1983), dans son travail sur le deuil et ses ratées, envisage une modalité mortifère du processus identificatoire. Pour survivre psychiquement à la perdition, l'enfant est contraint de désinvestir l'objet d'amour qui, absorbé dans un deuil indicible et empreint de culpabilité en mal d'élaboration, se détourne de lui. L'identification, qui prend les allures d'un emprunt, porte alors sur le sentiment de culpabilité inconscient de l'objet que l'enfant n'arrive plus à investir. Tel « un lien secret, témoin unique de ce qui reste des amours anciennes» (Cournut, 1983, 130), l'emprunt, ignoré 
comme emprunt, conserve cet amour ambivalent pour l'autre endeuillé. À son insu, l'emprunteur prend en charge quelque chose de la souffrance de l'autre.

Désormais, de nouvelles questions se posent mais auront à demeurer en suspens. D'une part, cette attraction du patient pour la fuite, dans son rapport aux hommes, remet-elle en chantier, perpétuel et constant, une perte, voire une défaite d'amour ou la précarité d'un lien avec un homme? La fuite provoque-t-elle répétitivement une perte, le fatum d'un désinvestissement?

Dans la mesure où le patient peut être rapproché des emprunteurs de Cournut (1983), cette perte appartiendrait moins à son histoire personnelle qu'à celle de la personne qu'il tenterait de réparer. Dans le portrait familial du patient qui a été plus accessible à ma rêverie, cette personne à réparer apparaît sous les traits de la mère d'accueil, peut-être soumise au(x) deuil(s) infligé(s) par la relation à son propre père. Cette personne apparaît aussi sous les traits de la mère biologique, dont il a été dit qu'elle souffrait d'un trouble de la personnalité sans que celui-ci ne soit rattaché à quelque histoire de vie difficile. La détresse de celle-là paraît aussi intense qu'elle resterait énigmatique et peu élaborée par l'entourage adulte du patient. Que la souffrance de la mère biologique manque à être pensée rend possible que le fils panse la mère, en lui empruntant ses deuils. Aussi, le patient qui, par rapport aux hommes, se sent comme un monstre, se sent-il ainsi à la place de la mère - à la place des mères qui, tour à tour, l'ont abandonné? Exprime-t-il là un indicible des mères, quelque chose de leur sauvagerie pulsionnelle à elles, dont l'élaboration leur serait aussi inabordable que la blessure, dans leur relation au père (ou plus largement, à l'homme, au parent ou à l'adulte), serait profonde? Et qu'en estil du père de l'enfant, cet homme disparu, dans l'engrenage des deuils?

Freud $(1921,194)$ précise que l'identification «a, entre autres, comme conséquence qu'on limite l'agression contre la personne à laquelle on s'est identifié, qu'on la ménage et qu'on lui apporte de l'aide». Alors, l'analyse de l'emprunt, et l'élaboration de l'oblation qui y est actuelle, risque-t-elle d'ouvrir la voie à l'expérience de mouvements pulsionnels inédits... et à une nouvelle possibilité d'éprouver la culpabilité? Cette fois, ce pourra être celle du patient qui parle - mais on n'en sera jamais certain.

\section{Une parole pour un temps autre}

Ce travail s'inscrit dans le cadre d'un atelier intitulé «Psyché et homophobie: questionner les interactions historiques et actuelles ». Ma présentation tire les éléments de ce titre vers un angle particulier, vers l'interrogation d'un 
temps actuel, voire intemporel, au sens psychanalytique, qui déterminerait l'écriture de l'histoire telle qu'elle est racontée. La tâche devient celle de favoriser l'ouverture des récits, dans lesquels de l'homosexualité (la sienne ou celle d'autrui) est à la fois identifiée et rejetée (par soi ou par autrui), sur une intensité qui n'a pas encore été éprouvée par le moi qui raconte, une intensité qui appellerait néanmoins le récit et qui résonnerait dans l'acte qui le transmet.

Ainsi, à travers une écoute à perte de vue pour des événements repérables chronologiquement, un temps autre se déporte jusqu'à nous. L'intensité muette, délayée dans les récits d'une visite dans le Village ou d'une scène d'intimidation, s'incarne éventuellement en des mots, nouveaux. Il y a transfert de celle-là dans la parole. Parole qui dit, à l'évidence. Mais aussi, parole qui fait vivre le doute d'amour, la détresse de l'abandon et la culpabilité-monstre, témoin d'une passion pour la mère et pour quelque autre objet perdu. Parole qui les porte, vivant.

Il est fréquent de tenter de contrer les phénomènes identifiables à l'homophobie par un travail de valorisation et de représentation de l'homosexualité. Il me semble néanmoins qu'à l'envers de la découverte de l'homosexualité (la sienne ou celle d'autrui) et du désarroi qui l'accompagne fréquemment, il faille peut-être envisager un désarroi à ne pas découvrir ou, encore, à ne pas se représenter suffisamment certains mouvements animant l'âme humaine. À l'envers de ce qui s'identifie comme homophobie, à la fois celle de l'environnement et celle dont on dit qu'elle est intériorisée, il faut peut-être envisager une infantilophobie, en tout cas un rejet apposé à des intensités infantiles, plus effrayantes et archaïques que l'homosexualité, mais qui seraient jugées «homosexuelles» par le moi. Ces intensités ressembleraient sûrement moins à quelque orientation ou identité sexuelles, qu'elles concerneraient les désastres d'amour et les excès passionnels qui sont suspendus dans l'impensé de l'être humain, dans son inconscient. Paradoxalement, l'être humain les aurait déjà, et pas encore, vécus. Désastres et excès restent à être imaginés, depuis le temps immémorial de sa rencontre avec un monde adulte, alors qu'il n'était qu'un enfant radicalement dépendant, voire à la merci de la souffrance de ce monde adulte.

L'enfant auquel s'intéresse la psychanalyse n'est pas uniquement l'enfant que l'adulte a été, l'enfant d'une enfance chronologiquement repérable, un enfant qui ne serait plus. L'enfant de la psychanalyse, que Scarfone (2010) après Laplanche (1999) ne manque pas de rappeler, est l'infans qui remue l'être humain au présent: l'infans, c'est-à-dire ce qui ne parle pas dans l'humain, l'autre-chose qui n'est pas doué de la parole, le désignifié qui ne 
cesse d'ébranler son être ainsi que les formes identitaires et narratives auxquelles il s'accroche, une condition d'ébranlement, contractée dans la rencontre avec l'autre-personne. Infantilophobie: peur de l'enfant, l'enfant qui n'a pas été, peur tout autant de ce qui n'a pas (en soi) été enfant, enfant qui ne sera jamais une fois pour toutes, enfant-spectre diffusé dans la parole et la pensée. Dans les limbes, selon la belle poésie de Pontalis (1998), cet enfant resterait, encore et toujours, à éprouver.

Avec la psychanalyse, s'engager sur le terrain de la lutte contre l'homophobie, ce n'est pas seulement repenser l'objet de ladite phobie. C'est aussi reconsidérer le projet même d'une lutte, hors de sa visée habituelle d'emprise sur une altérité hostile. C'est envisager une expérience de la force, la sienne et celle de l'autre, qui ne se confonde pas avec la mise en acte d'une volonté de puissance. "Vous êtes fort monsieur» avait répondu le patient à une des interprétations qui lui avait été communiquée. En séance, il était bien sûr fréquemment question, plus ou moins explicitement, de la force des hommes et de la sienne. Cette force violente, qui revêtait à certains moments un aspect homophobe, semblait s'exercer selon une loi du plus fort que le patient entretenait dans ses contre-attaques (sa contre-homophobie) ou à laquelle il réagissait souvent par la fuite. Or, cette force qu'il a supposé être la mienne, à ce moment bien précis dois-je ajouter, semble favoriser davantage son rapprochement que son retrait. Ce n'est plus une force par rapport à laquelle il aurait été faible, mais une force par rapport à laquelle il peut s'éprouver un peu plus largement, dans sa vulnérabilité humaine et ses désirs. Peut-être qu'à ce moment, il voit moins le monde comme une menace parce qu'une partie de lui, ou une partie de l'autre en lui, trouvant asile dans une pensée vivante, n'est plus condamnée au désespoir de détruire ${ }^{6}$ ? Comment comprendre cette (amorce de) resignification d'une force qui opprime l'autre, en une force qui permette davantage une proximité avec lui ? À ce sujet, je me réfère à Lévinas $(1974,271)$ lorsqu'il écrit :

Le vrai problème pour nous autres Occidentaux, ne consiste plus tant à récuser la violence qu'à nous interroger sur une lutte contre la violence qui - sans s'étioler dans la non-résistance au Mal - puisse éviter l'institution de la violence à partir de cette lutte même. Il faut reconsidérer le sens d'une certaine faiblesse humaine et ne plus voir dans la patience uniquement l'envers de la finitude ontologique de l'humain. Mais pour cela être patient soimême, sans demander la patience aux autres; et, pour cela, admettre une différence entre soi et les autres. 
Je considère qu'une écoute patiente de l'autre, une écoute sensible aux repères signifiants par lesquels s'exprime sa différence, une écoute qui (autant que possible) ne sait pas à l'avance ce qu'elle entendra, peut certes participer d'une lutte contre la violence qui évite d'instituer la violence à partir de cette lutte même. Dans ce sens, une certaine écoute de l'autre pourrait bien soutenir la transformation d'une expérience de la force comme pouvoir d'oppression, en une expérience de la force comme pouvoir de vitalité, une force qui ne serait allergique ni à l'épreuve de la fragilité humaine ni à celle d'une réceptivité pour l'autre, une force à l'accueil de ce qui vient.

De façon originale, Rolland $(1998,2006)$ souligne la fonction endoperceptive de la parole: ce serait elle qui lirait l'intensité inconsciente, et ajouterais-je, qui la lierait également. Ce serait elle qui en permettrait l'intégration éventuelle dans une langue habitée par le moi. Par le fait même, écouter autrui ce serait l'écouter à partir de la parole qui circule à l'intérieur de soi, entendre quelque chose de l'inconscient à partir du discours intérieur qui est le nôtre, bien que rendu possible par les paramètres de notre culture. Et lorsque l'autre à écouter agit plus qu'il ne parle, l'advenue de ce discours intérieur n'est que plus déterminante afin que l'autre, à son tour, puisse parler. Alors, c'est d'abord à l'intérieur de soi que doit s'incarner une parole liante, une parole, regardée par l'inconnu, rêvant là où ça n'a jamais été pensé.

C'est au nom de cet inconnu que j'espère une lutte contre l'homophobie qui puisse rester ouverte. Cela implique une disponibilité envers l'intense et l'inédit animant néanmoins, en creux, l'homosexualité identifiée et l'homophobie identifiable dans tant de trajectoires singulières. Intensité infantile qui, dans la mesure où elle n'a pas été suffisamment éprouvée, déterminerait un faire-violence autant qu'un subir-violence.

Anthony Bourgeault anthony.bourgeault@umontreal.ca

\section{Notes}

1. Je dois cette image de l'extraction à Scarfone (2007).

2. Dans la pensée de Lyotard (1988), la passibilité désigne la condition de l'être humain qui éprouve à partir de ce qu'il reçoit originairement de l'autre, l'être humain qui est saisi par un affect qui lui arrive d'abord, dans sa rencontre avec l'autre, comme ce qu'il n'a pas contrôlé.

3. Je remercie Anne-Louise Robichaud, psychologue-superviseure, pour l'inspiration clinique et théorique insufflée à ce suivi thérapeutique.

4. Le Village désigne un quartier montréalais où se regroupent des bars, des restaurants et des magasins principalement fréquentés par la communauté gaie et lesbienne. 
5. Roussillon $(1999,90)$ articule trois impératifs à l'idée de survivre: « ne pas se retirer de la relation, ne pas exercer de rétorsion agressive, et qualifier ce qu'il y a de potentialité créatrice et adaptative dans la destructivité [de l'autre] ». L'objet détruit, soit l'objet qui ne survit pas, tend ainsi à se détourner de la relation à l'autre humain. Également, il tend à ne pas répondre à l'appel au lien, qui serait contenu dans toute communication, en dépit du caractère hostile ou destructeur qu'elle peut manifester.

6. Parler d'un monstre et d'un danger est déjà différent que de faire le monstre et que de réagir au danger.

\section{Références}

AGAMBEN, G., 1998, Ce qu'il reste d'Auschwitz, Paris, Payot.

AULAGNIER, P., 1991, Temps de parole et temps de l'écoute, in Aulagnier, P., Un interprète en quête de sens, Paris, Petite bibliothèque Payot. 117-142.

BLEICHMAR, S., 2010, Paradoxes de la sexualité masculine, Paris, PUF.

BORILlO, D., 2003, Homophobie, in Éribon, D., Dictionnaire des cultures gays et lesbiennes, Paris, Larousse, 255.

COURNUT, J., 1983, D’un reste qui fait lien : à propos du sentiment inconscient de culpabilité emprunté, Nouvelle Revue de psychanalyse, 28, 129-150.

FREUD, S., 1916, Totem et tabou, Paris, PUF.

FREUD, S., 1919, Un enfant est battu, in Freud, S., Névrose, psychose et perversion, Paris, PUF, 219-243.

FREUD, S., 1921, Psychologie des foules et analyse du moi, in Freud, S., Essais de psychanalyse, Paris, Petite bibliothèque Payot, 129-242.

GANTHERET, F., 1992, Interpréter, Trans, 1, 95-110.

GANTHERET, F., 1996, Moi, monde, mots, Paris, Gallimard.

KLEIN, M., 1968a, L’amour, la culpabilité et le besoin de réparation, in Klein, M., Riviere, J., L'amour et la haine, Petite bibliothèque Payot, Paris. 83-164.

KLEIN, M., 1968b, Envie et gratitude et autres essais, Paris, Gallimard.

LAPLANCHE, J., 1999, Entre séduction et inspiration: l'homme, Paris, PUF.

LÉVINAS, E., 1974, Autrement qu'être, Paris, Kluwer Academic.

LYOTARD, J.-F., 1988, L'inhumain, Paris, Galilée.

PONTALIS, J.-B., 1988, Perdre de vue, Paris, Gallimard.

PONTALIS, J.-B., 1997, Ce temps qui ne passe pas, Paris, Gallimard.

PONTALIS, J.-B., 1998, L'enfant des limbes, Paris, Gallimard.

PONTALIS, J.-B., DE M'UZAN, M., 1977, Écrire, psychanalyser, écrire, Nouvelle Revue de psychanalyse, 16, 5-26.

ROLLAND, J.-C., 1998, Guérir du mal d'aimer, Paris, Gallimard.

ROLLAND, J.-C., 2006, Avant d'être celui qui parle, Paris, Gallimard.

ROUSSILLON, R., 1999, Agonie, clivage et symbolisation, Paris, PUF.

SCARFONE, D., 2000, Sexuel et actuel, in Widlöcher, D., Sexualité infantile et attachement, Paris, P.U.F., 147-167.

SCARFONE, D., 2007, Entre sens et présence, la répétition, Major lecture présentée au Congrès de l'Association psychanalytique internationale, Berlin.

SCARFONE, D., 2010, Dans le creux du transfert: l'analyste entre activité et passivité, Libres Cahiers pour la psychanalyse, 1 (23), 149-164.

SMIRNOFF, V., 1977, Épreuves, Nouvelle Revue de psychanalyse, 16, 195-202. 